\title{
Reconsideration of Vascular Selectivity of Dihydropyridine Calcium Antagonists: Comparison of Cardiovascular Profile of Mepirodipine, a Novel Dihydropyridine Consisting of a Single Stereoisomer with (+)-(S)-(S) Conformation, with Those of Nifedipine and Nicardipine
}

\author{
Shigeru MOTOMURA and Keitaro HASHIMOTO \\ Department of Pharmacology, Yamanashi Medical College. \\ Tamaho, Yamanashi 409-38, Japan \\ Accepted October 19,1989
}

\begin{abstract}
The coronary vasodilator effect and negative chronotropic, inotropic and dromotropic effects of mepirodipine, the single stereoisomer of a novel dihydropyridine $\mathrm{Ca}$ antagonist, were compared with those of nifedipine and nicardipine using canine isolated, blood-perfused heart preparations. Drugs were injected into each nutrient artery. In the sinoatrial node preparation, the dose producing a $15 \%$ decrease in sinoatria! rate was $1.3,6.5$ and $2.5 \mathrm{~kg}$ for mepirodipine, nifedipine and nicardipine, respectively. In the papillary muscle preparation, the dose causing a $50 \%$ decrease in developed tension was $44,6.5$ and $54 \mu \mathrm{kg}$ for mepirodipine, nifedipine and nicardipine, respectively. The dose causing a $50 \%$ increase in blood flow through the anterior septal artery was 0.26 .0 .18 and $2.0 \mu \mathrm{g}$ for mepirodipine, nifedipine and nicardipine, respectively, while the time required for return to half maximum at the above dose was 13.1, 1.8 and $4.1 \mathrm{~min}$, respectively. In the atrioventricular node preparation, the dose producing a $50 \%$ increase in $\mathrm{AH}$ interval was 1.6. 2.4 and $3.7 \mathrm{~kg}$ for mepirodipine, nifedipine and nicardipine, respectively. These results indicate that mepirodipine is a potent and long-lasting dihydropyridine $\mathrm{Ca}$ antagonist. whose vascular selectivity is highest against cardiac contractility, but less selective against sinoatrial node automaticity and atrioventricular nodal conduction, compared with nifedipine.
\end{abstract}

The tissue specificity of calcium (Ca) antagonists is still a subject of much interest: what properties of the $\mathrm{Ca}$ antagonists or tissues are responsible for vascular selectivity (1) ? Ca antagonists are more potent in producing vascular relaxation than in causing cardiac depressant effects expressed in negative inotropy, chronotropy and dromotropy $(2-5)$. Dihydropyridine $\mathrm{Ca}$ antagonists have higher vascular selectivity than non-dihydropyridines (2-7). The higher vascular selectivity of the dihydropyridine $\mathrm{Ca}$ antagonists may be explained by the fact that nitrendipine preferentially binds to the L-type Ca channels in the inactivated state, which is prompted by membrane depolarization, with more than 1000 times higher affinity than in the resting state $(8,9)$. However, it has been noticed that there are differences in the selectivity spectra of coronary vasodilator and cardiac depressant effects; non-dihydropyridine $\mathrm{Ca}$ antagonists such as verapamil and diltiazem are nearly equieffective on vasculature, sinoatrial (SA) node and atrioventricular (AV) node, but less effective on ventricular muscle contraction (3, 6). Even among dihydropyridine $\mathrm{Ca}$ antagonists, nicardipine and nimodipine are less vasoselective than nifedipine as compared with the negative chronotropy of the SA node automaticity and with the negative dromotropy of the AV node (6). Recently. Humphries and $O^{\prime}$ Conner (10) demonstrated that FPL 62129, a novel dihydropyridine $\mathrm{Ca}$ channel blocker, showed a much higher ac- 


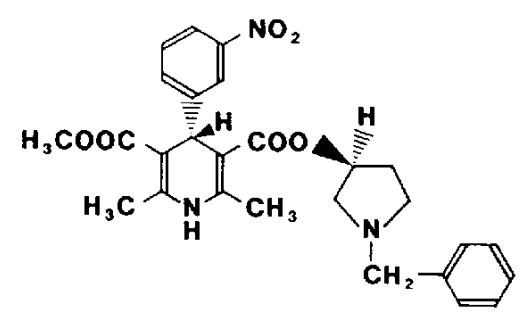

Mepirodipine $(+)-(S)-(S)$

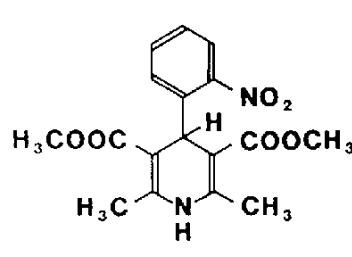

Nifedipine

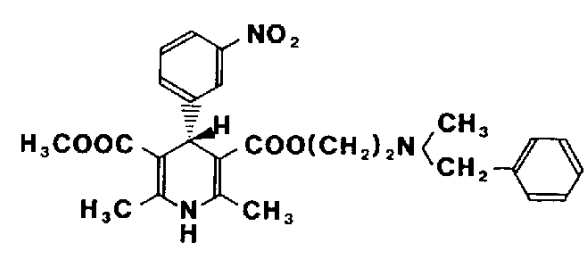

Nicardipine $(+)-(S)$

Fig. 1. Chemical structure of mepirodipine, a single stereoisomer with $(+)-(S)-(S)$ conformation, in comparison with nifedipirie and $(+)-(S)$ nicardipine.

tivity than nifedipine at the SA node and, to a slightly lesser extent, at the AV node, although both dihydropyridines have potent inhibitory effects on vascular smooth muscle. They suggested that the heterogeneity in the pharmacological properties of $\mathrm{Ca}$ antagonists should be reconsidered with respect to both vascular versus cardiac and intracardiac tissue selectivities. In other words, vasodilator effects should be compared with direct negative chronotropic and dromotropic effects on the $S A$ and $A V$ nodes, in addition to the negative inotropic effects, even among the dihydropyridines.

Recently, a novel dihydropyridine $\mathrm{Ca}$ antagonist, mepirodipine (YM-09730-5), which is a derivative of nicardipine and a single stereoisomer (+)-(S)-(S) conformation (Fig. $1)$, has been developed (11). It showed a potent long-lasting coronary vasodilator effect compared with the other three enantiomers. In the present experiments, we attempted to elucidate the cardiovascular profile of mepirodipine, by using the canine isolated, blood-perfused heart preparations in comparison with those of nifedipine and nicardipine. Using this information, the selectivity of the dihydropyridine $\mathrm{Ca}$ antagonists for coronary blood flow versus cardiac variables can be reconsidered.

\section{Materials and Methods}

The isolated heart preparations were obtained from mongrel dogs of either sex, weighing approximately $10 \mathrm{~kg}$, anesthetized with sodium pentobarbital $(30 \mathrm{mg} / \mathrm{kg}, i . v$.), given sodium heparin ( $500 \mathrm{U} / \mathrm{kg}$, i.v.) and exsanguinated. The heart was excised and plunged into cold Tyrode's solution kept at about $4^{\circ} \mathrm{C}$; then heart preparations were prepared.

The SA node preparation consisted of the entire right atrium, and the right coronary artery (RCA) was cannulated (12). The sinoatrial rate (SAR) of the SA node preparation was measured with a cardiotachograph (San-ei Instruments, 1321) triggered by bipolar atrial electrograms obtained from the SA node area.

The papillary muscle (PM) preparation consisted of the anterior papillary muscle of the right ventricle attached to the interventricular septum, and the anterior septal artery (ASA) was cannulated $(7,13,14)$. The PM preparation was electrically driven at a fixed rate of $2 \mathrm{~Hz}(120$ beats $/ \mathrm{min})$ by a stimulator (Dia Medical, DHM-226-3) and an isolation unit (Dia Medical, DPS-110) with rectangular pulses of $1-3 \mathrm{~V}$ (about $20 \%$ above the threshold voltage) at $5 \mathrm{msec}$ duration through 
bipolar silver-silver stimulating electrodes sutured onto the endocardium of the ventricular septum close to the base of the PM. Developed tension (DT) of the PM preloaded with the weight at which maximum tension developed was measured isometrically using a force displacement transducer (Dia Medical. DRM-T200) and an amplifier (Dia Medical. DRM-T20).

The AV node preparation consisted of the interventricular septum and the right atrium: and the posterior septal artery (PSA, the AV node artery), the ASA and the RCA were cannulated (15-17). The crista terminalis of the right atrium was electrically driven at a fixed rate of $2.5 \mathrm{~Hz}$ (150 stimuli/min) by a stimulator (Dia Medical, DHM-226-3) and an isolation unit (Dia Medical, DPS-110) with rectangular pulses of $1-3 \mathrm{~V}$ (about $20 \%$ above the threshold voltage) at $5 \mathrm{msec}$ duration through bipolar stimulating $\mathrm{Ag}-\mathrm{Ag}$ electrodes. Bipolar atrial (A) electrograms obtained from the right atrial free wall. His bundle $(H)$ electrograms from the His bundle region, and ventricular $(V)$ electrograms from the base of the anterior papillary muscle of the right ventricular septum were fed to an automatic AH interval meter (Dia Medical, DHM-2261), which individually measures $A V, A H$ and $\mathrm{HV}$ intervals with an analysis pitch of $1 \mathrm{msec}$ (17).

The SA node and PM preparations were placed in each double-wall glass jacket maintained at $38^{\circ} \mathrm{C}$ by circulating warm water. Both SA node and PM preparations were simultaneously cross-circulated through each cannulated artery with the heparinized arterial blood of the donor dog at a constant perfusion pressure of $120 \mathrm{mmHg}$ with a Cole-Parmer Masterflex peristaltic pump and a Starling pneumatic resistance placed parallel to the perfusion system. Venous blood from the preparations and excess blood passing through the pneumatic resistance were collected in a blood reservoir and returned to the donor dog through the jugular vein. The rate of blood flow (BF) through the ASA and RCA was measured with each electromagnetic flowmeter (Nihon Kohden, MVF-1100) using a 2-mm cannulating flow probe.

In another series of experiments, the AV node preparation was perfused through the three cannulated PSA, ASA and RCA at a constant perfusion pressure of $120 \mathrm{mmHg}$, as in the cross-circulation of the SA node and PM preparations. The BF through the PSA, ASA and RCA was continuosuly measured.

Adult mongrel dogs of either sex, weighing $14-23 \mathrm{~kg}$. were used as donor dogs, which were anesthetized initially with pentobarbital sodium, $30 \mathrm{mg} / \mathrm{kg}$. i.v. and given an additional 4-5 $\mathrm{mg} / \mathrm{kg}$ every hour. The animals received an initial dose of $500 \mathrm{U} / \mathrm{kg}$ of heparin sodium. followed by $200 \mathrm{U} / \mathrm{kg}$ every hour. Respiration was controlled using an animal respirator (Harvard Apparatus, model 607). Systemic blood pressure and mean arterial pressure at the femoral artery and heart rate triggered by the $\mathrm{R}$ wave of the lead II ECG were monitored continuously with a polygraph (San-ei Instruments, 361-6).

Drugs used were mepirodipine hydrochloride, YM-09730-5. [(3s)-1-benzyl-3-pyrrolidinyl methyl (4s)-2,6-dimethyl-4-(m-nitrophenyl) -1.4-dihydropyridine-3.5-dicarboxylate hydrochloride] (Yamanouchi Pharmac. Co., Tokyo, Japan), nicardipine hydrochloride (Yamanouchi) and nifedipine (Bayer AG. Leverkusen, F.R.G.). Mepirodipine and nicardipine were dissolved in water to give a concentration of $1 \mathrm{mg} / \mathrm{ml}$. Nifedipine was in an ampoule at a concentration of 100 $\mu \mathrm{g} / \mathrm{ml}$. The desired concentrations of the drugs were obtained by diluting the stock solution with $0.9 \%$ saline. A volume of $30 \mu l$ was injected into the nutrient artery in each preparation over a period of $4 \mathrm{sec}$ with a microsyringe (Terumo, Japan).

$1 D_{15}(S A R)$, the dose causing a $15 \%$ decrease in sinoatrial rate in the SA node preparation: $1 D_{50}$ (DT), the dose causing a $50 \%$ decrease in developed tension in the PM preparation: $E D_{50}(C V D)$. the dose causing a $50 \%$ increase in blood flow through the anterior septal artery measured at the peak responses: $\mathrm{RT} 1 / 2$ (BF), the time required for return to the half maximum after a bolus injection at each $E D_{50}$ (CVD), which indicates the duration of the coronary vasodilator effect; $1 D_{50}(A H)$. the dose causing a $50 \%$ prolongation of $\mathrm{AH}$ interval in the AV node preparation; and RT1/2 (AV). which indicates the duration of the negative dromotropic effect at each $I_{50}$ $(\mathrm{AH})$, were obtained from the dose-response 


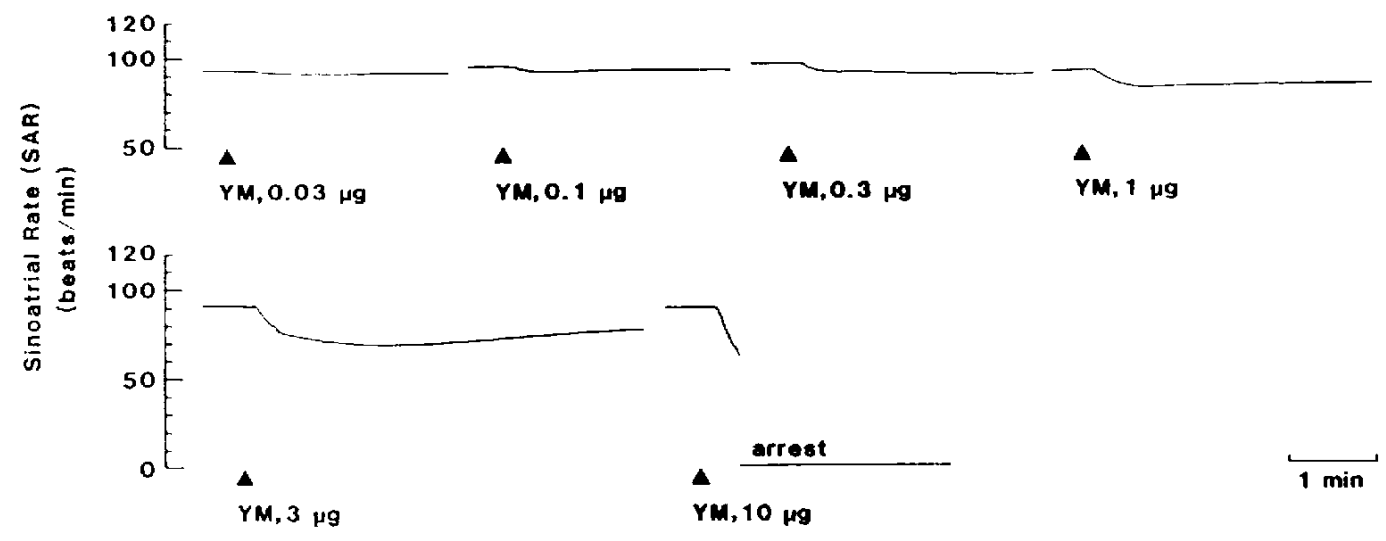

Fig. 2. An experiment to determine the effects of mepirodipine (YM) on sinoatrial rate of the sinoatrial node preparation. At $10 \mu \mathrm{g}$ of $\mathrm{YM}$, sinus arrest occurred.

curves.

\section{Results}

Effects on sinoatrial rate in the SA node preparation: In six SA node preparations, the basal SAR was $103 \pm 5$ beats/min. Mepirodıpine $(0.03-10 \mu \mathrm{g})$. nifedipine $(0.03-10 \mu \mathrm{g})$ and nicardipine $(0.1-10 \mu \mathrm{g})$ were injected into the RCA of the SA node preparation in a bolus fashion. An experiment showing the effects of mepirodipine on SAR is shown in Fig. 2. The SAR was dose-relatedly decreased and sinus arrest occurred at $10 \mu \mathrm{g}$ of mepirodipine. Dose-response curves for the negative chronotropic effects of mepirodipine, nifedipine and nicardipine are shown in Fig. 3. Sinus arrest was induced in all 6 SA node preparations by mepirodipine and in 4 of 6 preparations by nicardipine at $10 \mu \mathrm{g}$ of each drug, but not by nifedipine. The dose causing a $15 \%$ (approximately 15 beats $/ \mathrm{min}$ ) decrease in SAR obtained from each dose-response curve, $1 D_{15}(S A R)$, is shown in Table 1 . indicating that the potency of the negative chronotropic effect was in the order of mepirodipine, nicardipine and nifedipine.

Effects on developed tension and blood flow through ASA in the PM preparation: In six PM preparations, the basal DT was $2.9 \pm$ $0.2 \mathrm{~g}$ and $\mathrm{BF}$ through the ASA was $8.2 \pm 0.6$ $\mathrm{ml} / \mathrm{min}$. Mepirodipine $(0.03-10 / \mathrm{gg})$, nifedipine $(0.03-10 \mu \mathrm{g})$ and nicardipine $(0.3-100$ $\mu \mathrm{g})$ were injected into the ASA of the PM preparation in a bolus fashion. A typical experiment to determine the effects of

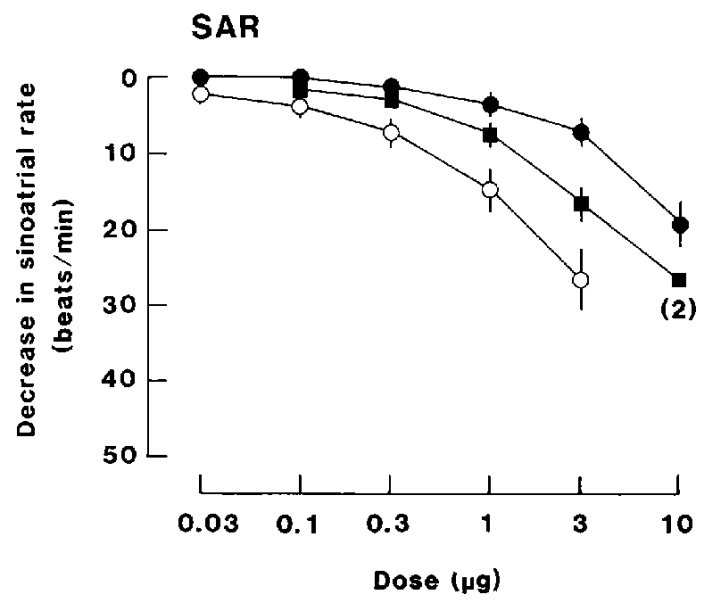

Fig. 3. Dose-response curves for negative chronotropic effects of mepirodipine (open circle), nifedipine (solid circle) and nicardipine (solid square). The values were expressed as the means $+S . E$. $(n=6$ for all drugs). At $10 \mu \mathrm{g}$. sinus arrest was induced in all 6 sinoatrial node preparations by mepirodipine and in 4 of 6 preparations by nicardipine. Nifedipine did not cause sinus arrest at all.

mepirodipine on DT and BF is shown in Fig. 4. DT was little affected but rather slightly increased at lower doses, and then doserelatedly but slightly decreased at higher doses of mepirodipine. BF was dose-relatedly increased in a wide dose range. Despite a bolus injection, increased BF markedly lasted. Figure 5 shows the dose-response curves for the negative inotropic effects (top) and for the coronary vasodilator effects (bottom) 
Table 1. Comparison of coronary vasodilator and negative chronotropic, inotropic and dramotropic effects of mepirodipine, nifedipine and nicardipine

\begin{tabular}{lcccccc}
\hline & $\begin{array}{c}\mathrm{ED}_{50}(\mathrm{CVD}) \\
(\mu \mathrm{g})\end{array}$ & $\begin{array}{c}\mathrm{ID}_{15}(\mathrm{SAR}) \\
(\mu \mathrm{g})\end{array}$ & $\begin{array}{c}\mathrm{ID}_{50}(\mathrm{DT}) \\
(\mu \mathrm{g})\end{array}$ & $\begin{array}{c}\mathrm{ID}_{50}(\mathrm{AH}) \\
(\mu \mathrm{g})\end{array}$ & $\begin{array}{c}\mathrm{RT} 1 / 2(\mathrm{BF}) \\
(\mathrm{min})\end{array}$ & $\begin{array}{c}\mathrm{RT1} / 2(\mathrm{AV}) \\
(\mathrm{min})\end{array}$ \\
\hline Mepirodipine & 0.26 & 1.3 & 44 & 1.6 & 13.1 & 8.5 \\
Nifedipine & 0.18 & 6.5 & 6.5 & 2.4 & 1.8 & 1.5 \\
Nicardipine & 2.0 & 2.5 & 54 & 3.7 & 4.1 & 3.2 \\
\hline
\end{tabular}

$E_{50}$ (CVD): the dose causing a $50 \%$ increase in blood flow through the anterior septal artery of the papillary muscle preparation, measured at the peak response. $I_{15}(S A R)$; the dose causing a $15 \%$ decrease in sinoatrial rate of the sinoatrial node preparation. $I_{50}(D T)$ : the dose causing a $50 \%$ decrease in developed tension of the papillary muscle. $\mathrm{ID}_{50}(\mathrm{AH})$ : the dose causing a $50 \%$ increase ir, $A H$ interval of the atrioventricular node preparation. RT1/2(BF): the time required for return to the half maximum increased blood flow after i.a. bolus injection at each $E D_{50}(C V D)$. RT1/2(AV): the time required for return to the half maximum increased atrioventricular conduction time after i.a. bolus injection at each $I D_{50}(A H)$. All values were obtained from the dose-response curves shown in Figs. 3, 5, 6 and 9.

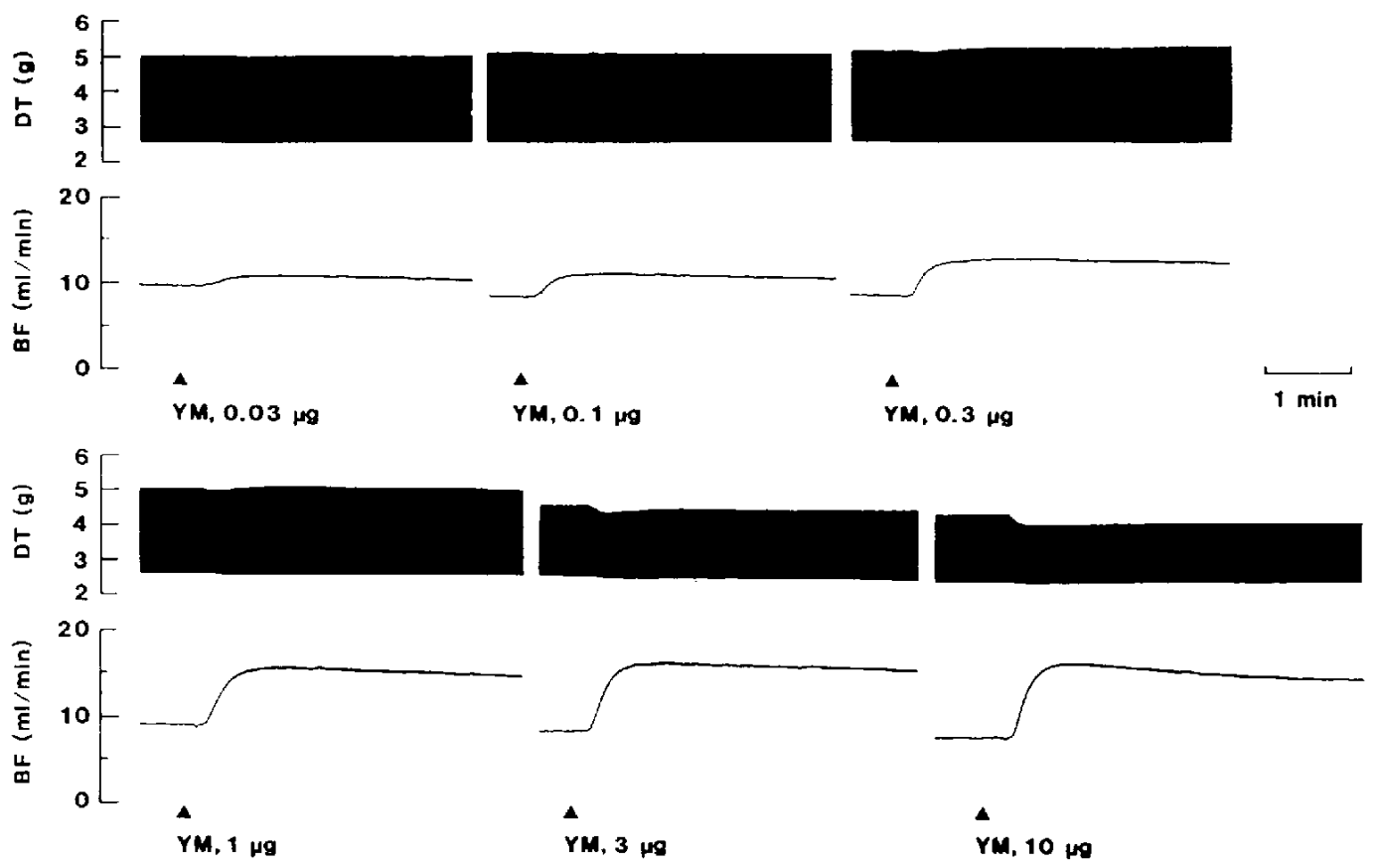

Fig. 4. A typical experiment of effects of mepirodipine (YM) on developed tension (DT) and blood flow (BF) through the anterior septal artery of the papillary muscle.

of mepirodipine, nifedipine and nicardipine. The decrease in DT was only about $20 \%$ even at $10 \mu \mathrm{g}$ of mepirodipine. At doses of 0.03 and $0.1 \mu \mathrm{g}$ of mepirodipine. $0.03 \mu \mathrm{g}$ of nifedipine and $0.3 \mu \mathrm{g}$ of nicardipine. DT was slightly increased. Increases in BF were measured at each peak response and expressed as percents of the basal BF before drug injection. $1 D_{50}$
(DT), the dose causing a $50 \%$ decrease in DT, is shown in Table 1, in which the value for mepirodipine was obtained by extrapolating the dose-response curve, indicating that the potency of the negative inotropic effect of mepirodipine was similar to that of nicardipine and that nifedipine was the most potent. On the other hand, $E D_{50}$ (CVD), the dose causing 

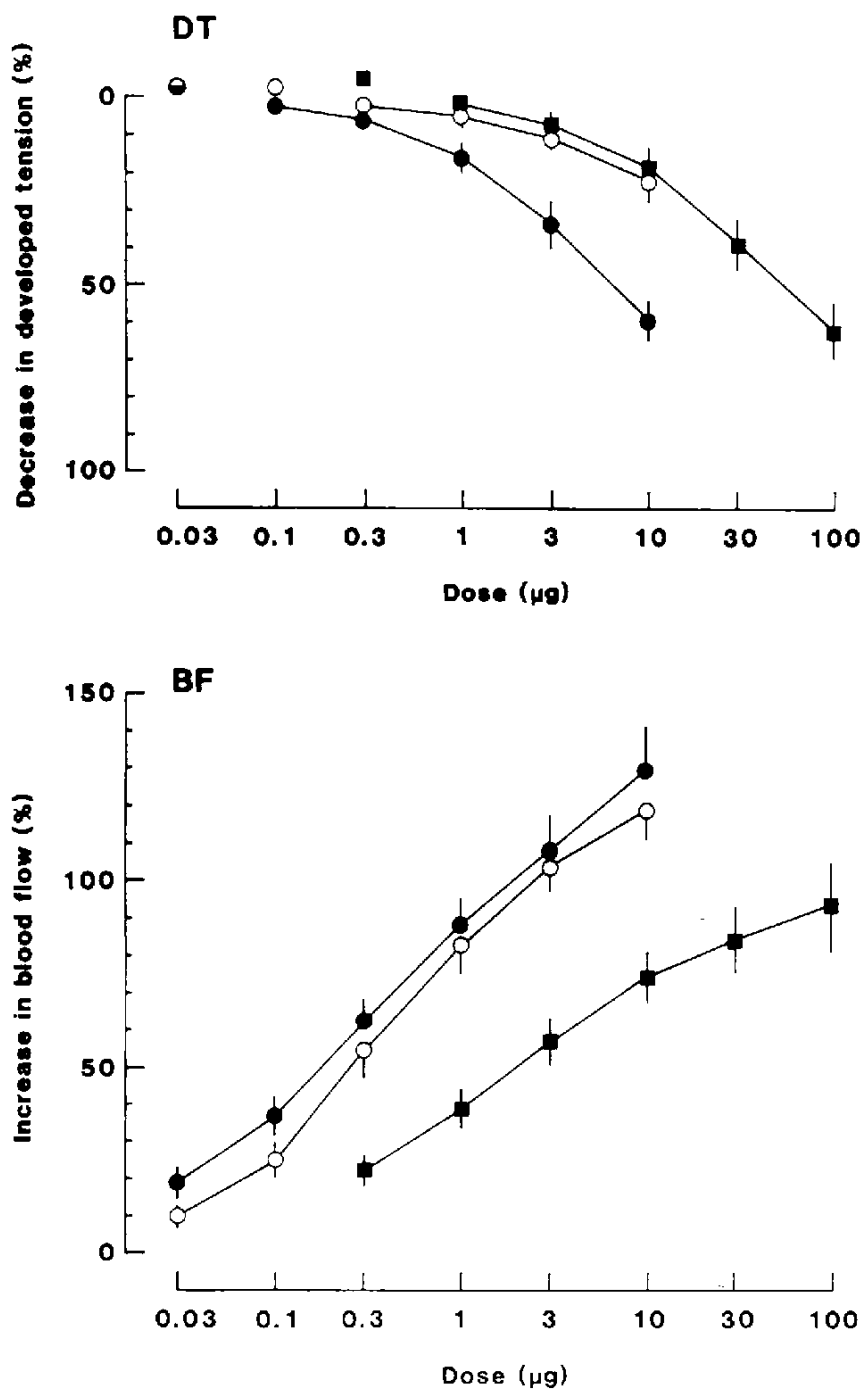

Fig. 5. Dose-response curves for negative inotropic (upper part, DT) and coronary vasodilator effects (lower part, BF) of mepirodipine (open circle). nifedipine (solid circle) and nicardipire (solid square). All points were obtained from 6 experiments (mean \pm S.E.).

a $50 \%$ increase in BF measured at the peak response, indicated that the potency of the vasodilator effect of mepirodipine was similar to that of nifedipine and that nicardipine was about 8 times less potent than the other two drugs (Table 1). In Fig. 6. RT1/2(BF), the time required for return to the half maximum increase in BF after a bolus injection, was doserelatedly increased in the cases of mepirodipine and nicardipine, but little affected in the case of nifedipine in a wide dose range.
RT1/2(BF) at each ED50(CVD), which indicates the duration of the same vasodilator effect as compared at the peak response, was extremely long for mepirodipine, intermediate for nicardipine, and quite short for nifedipine (Table 1).

Effects on AH interval in the AV node preparation: In eight $A V$ node preparations, the basal AV conduction time (AVCT). AH interval and $H V$ interval were $129 \pm 6 \mathrm{msec}, 95 \pm 5$ msec and $34 \pm 2 \mathrm{msec}$, respectively. Me- 


\section{RT $1 / 2$ (BF)}

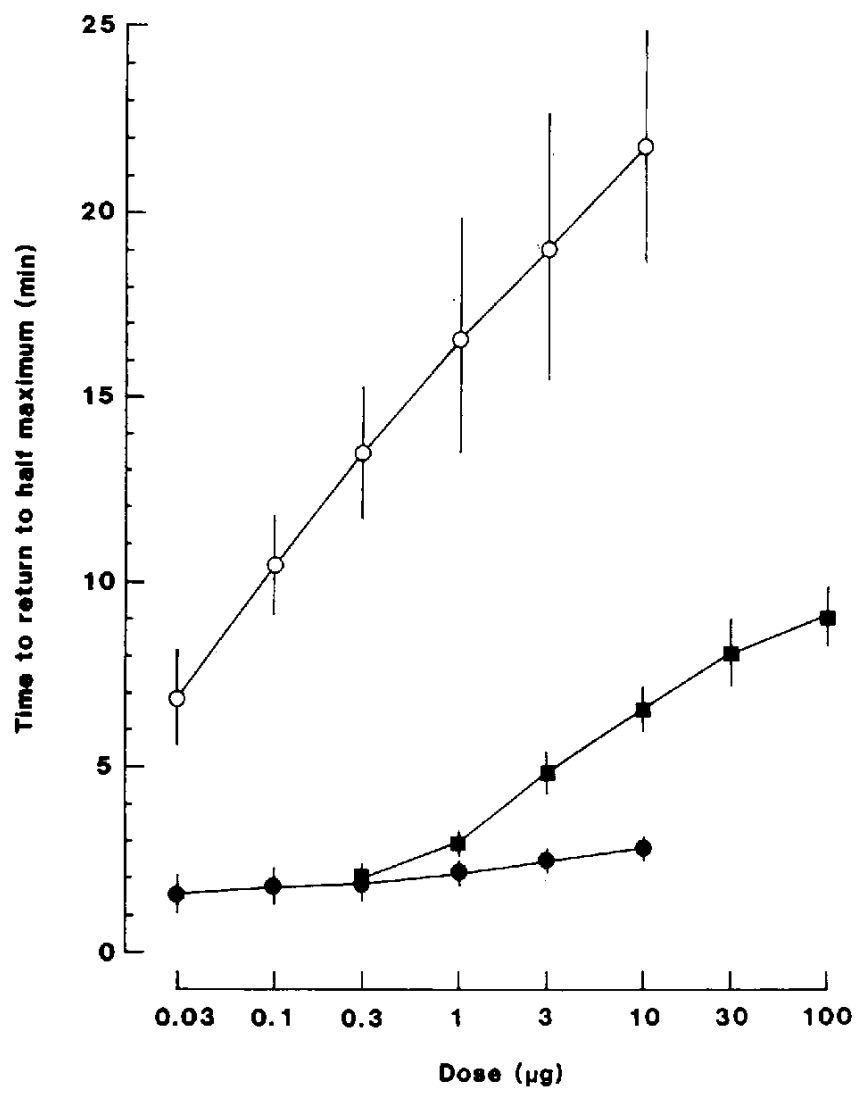

Fig. 6. Duration of coronary vasodilator effect of mepirodipine (open circle). nifedipine (solid circle) and nicardipine (solid square). RT1/2 (BF): time required for return to half maximum increase in blood flow after i.a. bolus injection (min). All points were obtained from 6 experiments (mean $\pm S$.E.).

pirodipine $(0.03-3 \mu \mathrm{g})$, nifedipine $(0.03-3$ $\mu \mathrm{g})$ and nicardipine $(0.1-3 \mu \mathrm{g})$ were injected into the PSA (the AV node artery) of the AV node preparation. A typical experiment to determine the effects of mepirodipine $(0.3 \mu \mathrm{g})$ injected into the PSA on atrial (A). Hisbundle $(\mathrm{H})$ and ventricular $(\mathrm{V})$ bipolar electrograms is shown in Fig. 7. The configuration of each electrogram was not changed by the PSA injection of mepirodipine. The interval between $A$ and $H$ electrograms was increased, while the interval between $H$ and $V$ electrograms was unaffected. Effects of mepirodipine (0.1-1 $\mu \mathrm{g})$ injected into the PSA on AVCT, AH and HV intervals are shown in Fig. 8; each value was obtained by the electrograms shown in Fig. 7 with an $\mathrm{AH}$ interval meter. The $\mathrm{AH}$ interval was dose-relatedly increased, but the HV interval was not affected by the PSA injection of mepirodipine. The prolongation of AVCT was entirely due to an increase in the $\mathrm{AH}$ interval. The effects on AVCT and $\mathrm{AH}$ interval were long-lasting even after bolus injections. When mepirodipine was injected into the ASA, which mainly perfuses the intraventricular (His-Furkinje-Ventricular muscle) conduction system, neither HV nor AH interval (i.e., AVCT) was affected (not shown). The upper part of Fig. 9 shows dose-response curves for the negative dromotropic effects of mepirodipine, nifedipine and nicardipine on AH interval. Second degree AV conduction block (AVB) was produced in 6 of 8 AV node preparations by mepirodipine, in 2 of 4 by nifedipine and in 1 of 4 by nicardipine at $3 \mu \mathrm{g}$ 
CONTROL

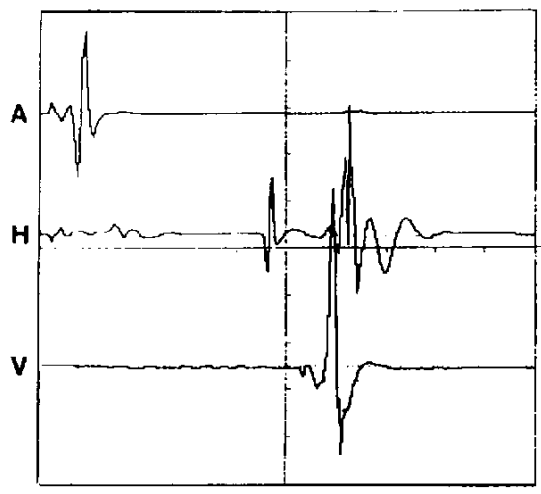

YM, $0.3 \mu g$ (PSA)

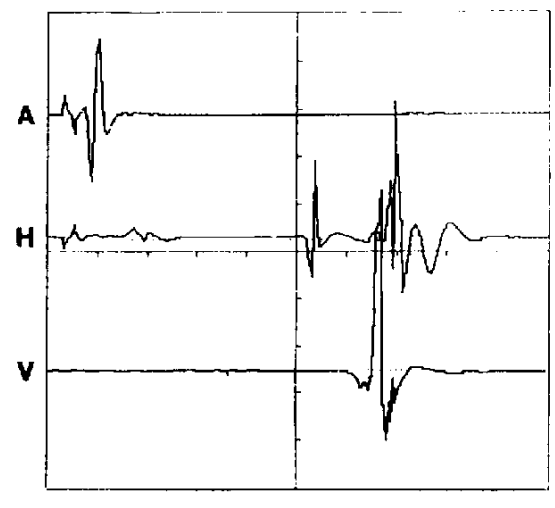

$50 \mathrm{msec}$

Fig. 7. Bipolar electrograms recorded from the right atriurn $(A)$, the His bundle $(H)$ and the ventricular septum $(V)$ in the atrioventricular node preparation. Configuration of each electrogram was unchanged by an injection of $0.3 \mu \mathrm{g}$ of inepirodipine (YM) in the posterior septal artery (PSA), but the interval between $A$ and $H$ electrograms was prolonged, while the interva! between $H$ and $V$ electrograms was unaffected.

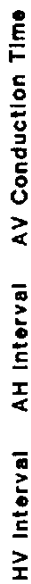

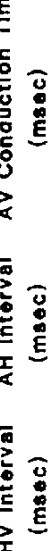

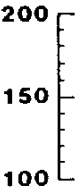
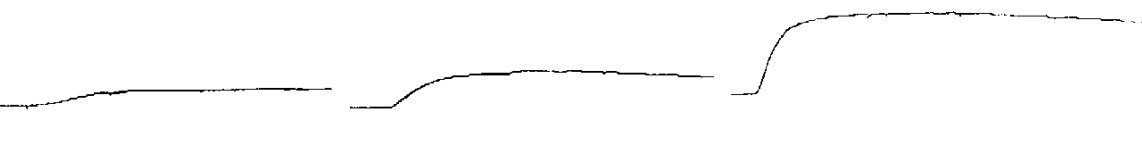

100
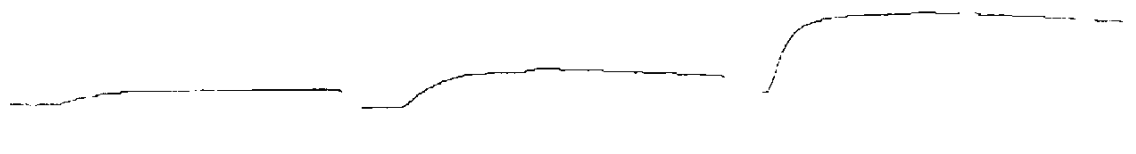

${ }^{50} E$

YM, 0.1 $\mathbf{\mu g}$

YM, $0.3 \mu g$

$\mathbf{Y M}, 1 \mathrm{mg}$

Fig. 8. A typical experiment to determine the effects of mepirodipine ( $Y M$ ) injected into the posterior septal artery on atrioventricular ( $A . V$ ) conduction time (top). $\mathrm{AH}$ (middle) and HV (bottom) intervals in the $A V$ node preparation. The values of $A V$ conduction time, $A H$ and $H V$ intervals were obtained by measuring automatically each interval between the electrograms shown in Fig. 7 with the AH interval meter.

of each drug. $I_{50}(A H)$, the dose causing a $50 \%$ increase (approximately $47 \mathrm{msec}$ ) in the $\mathrm{AH}$ interval, indicated that the potency of the negative dromotropic effect was in the order of mepirodipine, nifedipine and nicardipine. as shown in Table 1. The lower part of Fig. 9 shows that $\mathrm{RT} 1 / 2(\mathrm{AV})$, the time required for return to the half maximum increase in AVCT, i.e.. AH interval, was dose-relatedly prolonged after mepirodipine and, to the lesser extent, after nicardipine, but little changed after nifedipine. RT1/2(AV) at each $1 D_{50}(A H)$. shown in Table 1. indicated the long-lasting negative dromotropic effect of mepirodipine compared with those of nicardipine and nifedipine.

Selectivity for coronary blood flow versus cardiac variables: As shown in Table 2, the 
ratio of the negative inotropic versus coronary vasodilator effects, $I D_{50}(D T) / E D_{50}$ (CVD).
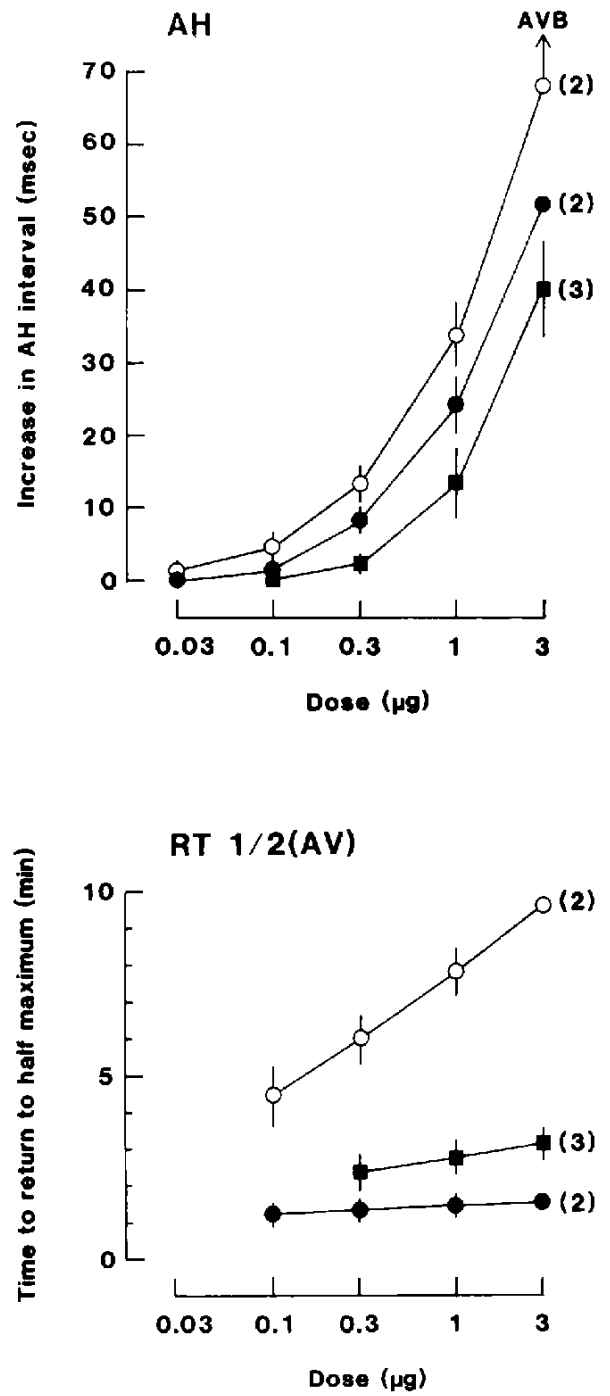

Fig. 9. Dose-response curves for negative dromotropic effects (upper part) and druration of the effects (lower part) of mepirodipine (open circle). nifedipine (solid circle) and nicardipine (solid square). RT1/2 (AV): time required for return to half maximum increase in atrioventricular (AV) conduction time after i.a. bolus injection (min). The values were expressed as the means \pm S.E. $(n=8$ for mepirodipine and $n=4$ for nifedipine and nicardipine). At $3 \mu \mathrm{g}$, second degree AV conduction block (AVB) was produced in 6 of $8 \mathrm{AV}$ node preparations by mepirodipine. in 2 of 4 preparations by nifedipine. and in 1 of 4 preparations by nicardipine. indicated that the three dihydropyridine $\mathrm{Ca}$ antagonists had extremely high coronary vascular selectivity compared with the negative inotropic effect and that mepirodipine had the highest selectivity, being about $4-5$ times more selective than nifedipine and nicardipine. On the other hand, the ratio of the negative chronotropic versus coronary vasodilator effects, $I D_{15}$ (SAR)/ED 50 (CVD), and the ratio of the megative dromotropic versus coronary vasodilator effects, $\mid D_{50}(A H) / E_{50}(C V D)$, indicated that nifedipine had the highest coronary vascular selectivity and that mepirodipine and in particular nicardipine were less vasoselective compared with both the SA and AV node functions among the three dihydropyridine $\mathrm{Ca}$ antagonists.

\section{Discussion}

Mepirodipine is a derivative of nicardipine and a single stereoisomer with $(+)-(S)-(S)$ conformation (Fig. 1), whose $K_{i}$ value for inhibition of $\left[{ }^{3} \mathrm{H}\right]$ nitrendipine binding to rat brain cortex homogenate membranes was very markedly smaller, $0.205 \mathrm{nM}$, than those of the other three enantiomers $(3.09 \mathrm{nM}$ for $(+)-(S)-(R), 49.6 n M$ for $(-)-(R)-(S)$ and $14.3 \mathrm{nM}$ for $(-)-(R)-(R))(11)$. The order of $K_{i} l$ values of the four enantiomers well-correlated to the potency order of their coronary vasodilator effects (11). In the same study. the $K_{i}$ value of nifedipine was $4.65 \mathrm{nM}$ and those of $(+)-(S)$ and $(-)-(R)$ nicardipine were $0.5 \mathrm{nM}$ and $7.11 \mathrm{nM}$, respectively. In the present experiments, however, the coronary vasodilator effect of mepirodipine was almost the same as that of nifedipine and was approximately 8 times more potent than that of nicardipine, when compared at the ceak response $\left[E D_{50}\right.$ (CVD)] (Table 1$)$. The $\mathrm{ED}_{50}$ (CVD) values of nifedipine and nicardipine in the present study were almost the same as those obtained with the same procedure in our previous study (7) and that of another group (6). On the other hand, the duration of the coronary vasodilator effect of mepirodipine was extremely long compared with those of nifedipine and nicardipine in the present experiments (Fig. 6 and Table 1). Since the duration of the coronary vasodilator effect of mepirodipine was longer than those of the other three enantiomers, the coronary vaso- 
Table 2. Selectivity for coronary blood flow versus cardiac variables: Ratio of coronary vasodilator effects versus negative chronotropic, inotropic and dromotropic effects of mepirodipine, nifedipine and nicardipine

\begin{tabular}{lccc}
\hline & $1 D_{15}(\mathrm{SAR}) / \mathrm{ED}_{50}(\mathrm{CVD})$ & $\mathrm{ID}_{50}(\mathrm{DT}) / \mathrm{ED}_{50}(\mathrm{CVD})$ & $\mathrm{ID}_{50}(\mathrm{AH}) / \mathrm{ED}_{60}(\mathrm{CVD})$ \\
Mepirodipine & 5 & 169 & 6 \\
Nifedipine & 36 & 36 & 13 \\
Nicardipine & 1 & 27 & 2 \\
\hline
\end{tabular}

Abbreviations are the same as those in Table 1.

dilator activity was estimated by the area under the increased blood flow curve (AUC) of four enantiomers (11). The AUC of meDirodipine was approximately 20-200 times larger than those of the other three enantiomers, suggesting that the $\mathrm{K}_{\mathbf{1}}$ values highly correlated to the AUC rather than the $E_{100}$ at the peak response (11). Although the AUC was not obtained in the present study, the values obtained from both $E D_{50}$ (CVD) and RT1/2(BF) showed a good correlation between the coronary vasodilator effects and the $K_{i}$ values. These previous results and the present ones suggest that the coronary vasodilator effects should be assessed from both the peak response and the duration, but not from only the former, and that the coronary vasodilator effects well reflect the affinity of the dihydropyridine $\mathrm{Ca}$ antagonists for the Ca channels to which nitrendipine bound.

The present experiments showed that mepirodipine had the highest vascular selectivity expressed as the ratio of coronary vasodilator versus negative inotropic effects, $1 D_{50}(D T) / E D_{50}$ (CVD). In the previous study (7), the $I D_{50}$ (DT)/ED 50 (CVD) of nitrendipine was calculated to be approximately 41 , which was higher than those of nifedipine and nicardipine, even so mepirodipine was about 4 times more vasoselective than nitrendipine. It is certain that mepirodipine, a single stereoisomer with (+)-(S)-(S) conformation. scarcely depressed cardiac contractility even when intraarterially administered. In this connection, it is of interest that amongst the dihydropyridines, there are $\mathrm{Ca}$ channel activators such as Bay k 8644 and Compound 202-791 whose stereoisomers showed strictly opposite actions $(18,19)$. Hof et al. (18) reported that the (-)-isomer of Compound 202-791 was a pure inhibitor of $\mathrm{Ca}$ entry, whereas the $(+)$ isomer enhanced $\mathrm{Ca}$ entry into cells. $\mathrm{Ac}$ - cording to Wei et al. (19), (t)-(R)-Bay k 8644 and $(-)-(R)-202-791$ inhibited the responses of rat tail artery to $\mathrm{KCl}$-induced depolarization. On the other hand, $(-)-(S)$ Bay k 8644 and (+)-(S)-202-791 induced and potentiated the responses to $\mathrm{KCl}$ depolarization at low concentrations, but at high concentrations, they relaxed the tissue contracted maximally at lower concentration of the same drugs (19). These reports prompted us to elucidate whether the enantiomers of mepirodipine have a $\mathrm{Ca}$ channel activating property, since mepirodipine is a single stereoisomer with (+)-(S) - (S) conformation (11). However, the four enantiomers of mepirodipine were all $\mathrm{Ca}$ antagonists, among which mepirodipine is the most potent (11). Thus, although the sparse negative inotropic effect could not be explained by its stereoselectively. mepirodipine may be useful for research on the steric structure of $\mathrm{Ca}$ channels.

In contrast to the sparse negative inotropic effect, the doses of mepirodipine for suppressing SA node automaticity and AV nodal conduction impairment were less separated from the coronary vasodilating dose. The least vascular selectivity compared with the negative chronotropic and dromotropic effects was observed for nicardipine among the three dihydropyridine $\mathrm{Ca}$ antagonists used in the present study (Table 1). Meanwhile, the doses of nifedipine for the negative inotropic, chronotropic and, to a lesser extent, dromotropic effects were almost equally separated from that of the coronary vasodilator effect (Table 1). Taira (6) showed that there are differences in selectivity spectra even among dihydropyridine $\mathrm{Ca}$ antagonists, in which nifedioine and nitrendipine are the most selective for coronary blood flow versus any of the other cardiac variables, but nicardipine and nimodipine are not highly selective in the 
coronary vasculature compared with their negative chronotropic and dromotropic effects. Similarly, Hof et al. $(20,21)$ suggested that the negative chronotropic effect of $P Y$ 108-068 and PN 200-110 could separated from the negative inotropic effect. Wada et al. (22) also showed that PY 108-068 and PN 200-110 had a high-degree separation of the negative inotropic effect, but small-degree separations of the negative chronotropic and dromotropic effects from the coronary vasodilator effect, although the negative dromotropic effect of PN 200-110 was moderately separated. Recently, Humphries and $\mathrm{O}^{\prime} \mathrm{Con}$ nor (10) showed that FPL 62129 selectively suppressed heart rate and AV nodal conduction, but had little effect on ventricular contractility, while nifedipine less selectively suppressed all intra-cardiac variables, when compared at the doses causing the same vasodilator effects (decreasing the total peripheral resistance). These results suggest that vascular selectivity of compounds should be reconsidered in comparison with their effects on the SA and AV nodal functions in addition to that on the cardiac contractility. even among the dihydropyridines.

The mechanisms by which the intra-cardiac tissue selectivity of $\mathrm{Ca}$ antagonists develops are still unknown. Differences in the physicochemical properties of the $\mathrm{Ca}$ antagonists and/or electrophysiological properties of the individual cardiac tissues have been proposed. Among the dihydropyridine $\mathrm{Ca}$ antagonists, nifedipine and nitrendipine are highly lipophilic, but mepirodipine and nicardipine are water-soluble. The pKa values are 7.52 for mepirodipine and 7.28 for nicardipine, respectively. The non-dihydropyridines, verapamil and diltiazem, are entirely ionized in physiological solution. Thus, water-soluble Ca antagonists, which sparsely enter into the cell through the lipid cell membrane, might preferentially suppress SA and AV nodal cells. Electrophysiologically, the SA and AV nodal cells have less negative resting membrane (maximal diastolic) potential than those of ventricular muscles. As mentioned earlier, the binding affinity of nitrendipine is prompted by membrane depolarization (8, 9). Thus both the physicochemical (water-soluble) property of the $\mathrm{Ca}$ antagonists and the electrophysiolo- gical (less negative membrane potential) property of the SA and AV nodal cells could be related to the intra-cardiac tissue selectivity of the dihydropyridine $\mathrm{Ca}$ antagonists.

In conclusion, mepirodipine is a potent and long-lasting dihydropyridine $\mathrm{Ca}$ antagonist with unique selectivity spectra; the coronary vasodilator effect is highly (approximately 4 times those of nifedipine and nitrendipine) selective compared with the negative inotropic effect, but less (approximately one third to one seventh of that of nifedipine) selective compared with the negative chronotropic and dromotropic effects. Thus, the present results suggest that the mechanism of vascular selectivity of the dihydropyridine $\mathrm{Ca}$ antagonists is not simple and that their vasodilator effect should be extensively compared with the negative chronotropic and dromotropic effects on both the SA and AV nodes, in addition to the negative inotropic effect. Nonetheless, mepirodipine could be useful for the treatment of hypertension without inducing cardiac failure, but effects on AV nodal conduction may occur.

\section{References}

1 Godfraind, T., More!, N. and Wibo, M.: Tissue specificity of dihydropyridine-type calcium antagonists in human isolated tissues. Trends Pharmacol. Sci. 9, 37-39 (1988)

2 Fleckenstein, A.: Specific pharmacology of calcium in myocardium. cardiac pacemakers, and vascular smooth muscle. Annu. Rev. Pharmacol. Toxicol. 17, 149-166 (1977)

3 Taira, N.: Effects of diltiazem and other calciumantagonists on cardiac functions and coronary blood flow as assessed in blood-perfused dogheart preparations. In New Drug Therapy with a Calcium Antagonist, Diltiazem Hakore Symposium 78. Edited by Bing. R.J., p. 91-103, Excerpta Medica, Amsterdam and Princeton (1979)

4 Henry, P.D.: Comparative pharmacology of calcium antagonists: Nifedipine. verapamil and diltiazem. Am. J. Cardiol. 46, 1047-1058 (1980)

5 Millard, R.W., Grupp, G., Grupp. I.L., DiSalvo, J., DePover, A. and Schwartz, A.: Chronotropic, inctropic, and vasodilator actions of diltiazem, nifedipine, and verapamil: A comparative study of physiological responses and membrane receptor activity. Circ. Res. 52, Supp. 1, I 29-1 39 (1983)

6 Taira, N.: Differences in cardiovascular profile 
among calcium antagonists. Am. J. Cardiol. 59, 24B-29B (1387)

7 Motomura, S., Hashimoto, K. and Hashimoto, K.: Effects of Bay $k 8644$ on the coronary vascular selectivity of the dihydropyridine Ca antagonists in the canine isolated, blood-perfused papillary muscle preparation. J. Cardiovasc. Pharmaco!. 10, 627-635 (1987)

8 Bean, B.P.: Nitrendipine block of cardiac calcium channels: High-affinity binding to the inactivated state. Proc. Natl. Acad. Sci. U.S.A. 81, 63886392 (1984)

9 Sanguinetti, M.C. and Kass, R.S.: Voltagedependent block of calcium channel current in the calf cardiac Purkinje fiber by dihydropyridine caicium chanrie! antagonists. Circ. Res. 55, 336348 (1984)

10 Humphries, R.G. and $0^{\circ}$ Connor, S.E.: Cardiovascular profile of FPL 62129, a novel dihydropyridine calcium channel blocker, in anaesthetized dogs: A comparison with nifedipine. J. Cardiovasc. Pharmacol. 11, 332-338 (1988)

11 Tamazawa, K., Arima, H., Kojima, T., Isomura, Y., Okada, M., Fujita, S., Furuya, T., Takenaka, T.r Inagaki, $\mathrm{O}$. and Terai, M.: Stereoselectivity of a potent calcium antagonist, 1-benzyl-3-pyrro!idinyl methyl 2.6-dimethyl-4-(m-nitrophenyl)1,4-dihydropyridine-3,5-dicarboxylate. J. Med. Chem. 29, 2504-2511 (1986)

12 Kubota, K, and Hashimoto, K.: Selective stimulation of the parasympathetic preganglionic nerve fibres in the excised and blood-perfused SA node preparation of the dog. Naunyn Schmiedebergs Arch. Pharmacol. 278, 135-150 (1973)

13 Endoh, M. and Hashimoto, K.: Pharmacological evidence of autonomic nerve activities in canine papillary muscle. Am. 1. Physiol. 218, 14591463 (1970)

14 Himori, N., Ono, H. and Taira, N.: Simultaneous assessment of effects of coronary vasodilators on the coronary blaod flow and the myocardial contractility by using the blood-perfused canine papillary muscle. Japan. J. Pharmacol. 26, 427435 (1976)
15 Hashimoto, K., lijima, T., Hashimoto, K. and Taira, N.: The isolated and cross-circulated AV node preparation of the dog. Tohoku J. Exp. Med. 107, 263-275 (1972)

16 Motomura, S., lij!ma, T., Taira, N. and Hashimoto, K.: Effects of neurotransmitters injected into the posterior and the anterior septal artery on the automaticity of the atrioventricular junctional area of the dog heart. Circ. Res. 37, 146-155 (1975)

17 Motomura, S. and Hashimoto, K.: Histamine $\mathrm{H}_{2}$ receptor mediated positive dromotropic effect in the canine atrioventricular node. Japan. J. Pharmacol. 49, 325-335 (1989)

18 Hof, R.P., Ruegg, U.T., Hof, A. and Vogel, A.: Stereoselectivity at the calcium channel: Opposite action of the enantiomers of a 1.4dihydropyridine. J. Cardiovasc. Pharmacol. 7, 689-693 (1985)

19 Wei, X.Y., Luchowski, E.M., Rutledge, A., Su, C.M. and Triggle, D.J.: Pharmacologic and radioligand binding analysis of the actions of 1,4-dihydropyridine activator-antagonist pairs in smooth muscle. J. Pharmacol. Exp. Ther. 239, 144-153 (1986)

20 Hof, R.P., Hof, A. and Neumann, P.: Effects of PY 108-068, a new calcium antagonist, on general hemodynamics and regional blood flow in anesthetized cats: A comparison with nifedipine. J. Cardiovasc. Pharmacol. 4, 352-362 (1982)

21 Hof, R.P., Scholtysik, G., Loutzenhiser, R., Vuorela, H.J. and Neumann, P.: PN 200-110, a rlew calcium antagonist: Electrophysiological, inotropic, and chronotropic effects on guinea pig myocardial tissue and effects on contraction and calcium uptake of rabbit aorta. J. Cardiovasc. Pharmacol. 6, 399-406 (1984)

22 Wada, Y., Satoh, K. and Taira, N.: Separation of the coronary vasodilator from the cardiac effects of PN 200-110, a new dihydropyridine calcium antagonist, in the dog heart. J. Cardiovasc. Pharmacol. 7, 190-196 (1985) 